\title{
Verification of exposure to Novichok nerve agents utilizing a semitargeted human butyrylcholinesterase nonapeptide assay
}

Daan Noort*, Alex Fidder, Debora van der Riet-van Oeveren, Ruud Busker and Marcel J. van der Schans

TNO - CBRN Protection, PO Box 45, 2280AA Rijswijk, The Netherlands

\section{Table of contents:}

Figure S1. MS/HRMS spectra of the single $\mathrm{m} / \mathrm{z} 970.4394$ (upper) and double $\mathrm{m} / \mathrm{z} 485.7233$ (lower) protonated molecule of phosphylated nonapeptide derived after pepsin digestion of $\mathrm{HuBuChE}$ isolated from plasma exposed to A-230.

Figure S2. Lower region (m/z 70-230) MS/HRMS spectra of the single m/z 970.4394 (upper) and double $\mathrm{m} / \mathrm{z} 485.7233$ (lower) protonated molecule of phosphylated nonapeptide derived after pepsin digestion of $\mathrm{HuBuChE}$ isolated from plasma exposed to A-230.

Figure S3. MS/HRMS spectra of the single $\mathrm{m} / \mathrm{z} 986.4343$ (upper) and double $\mathrm{m} / \mathrm{z} 493.7208$ (lower) protonated molecule of phosphylated nonapeptide derived after pepsin digestion of $\mathrm{HuBuChE}$ isolated from plasma exposed to A-232.

Figure S4. Lower region (m/z 70-230) MS/HRMS spectra of the single m/z 986.4343 (upper) and double $\mathrm{m} / \mathrm{z} 493.7208$ (lower) protonated molecule of phosphylated nonapeptide derived after pepsin digestion of $\mathrm{HuBuChE}$ isolated from plasma exposed to A-232.

Figure S5. MS/HRMS spectra of the single m/z 1000.4500 (upper) and double m/z 500.7286 (lower) protonated molecule of phosphylated nonapeptide derived after pepsin digestion of $\mathrm{HuBuChE}$ isolated from plasma exposed to A-234.

Figure S6. Lower region (m/z 70-230) MS/HRMS spectra of the single m/z 1000.4500 (upper) and double $\mathrm{m} / \mathrm{z} 500.7286$ (lower) protonated molecule of phosphylated nonapeptide derived after pepsin digestion of $\mathrm{HuBuChE}$ isolated from plasma exposed to A-234. 


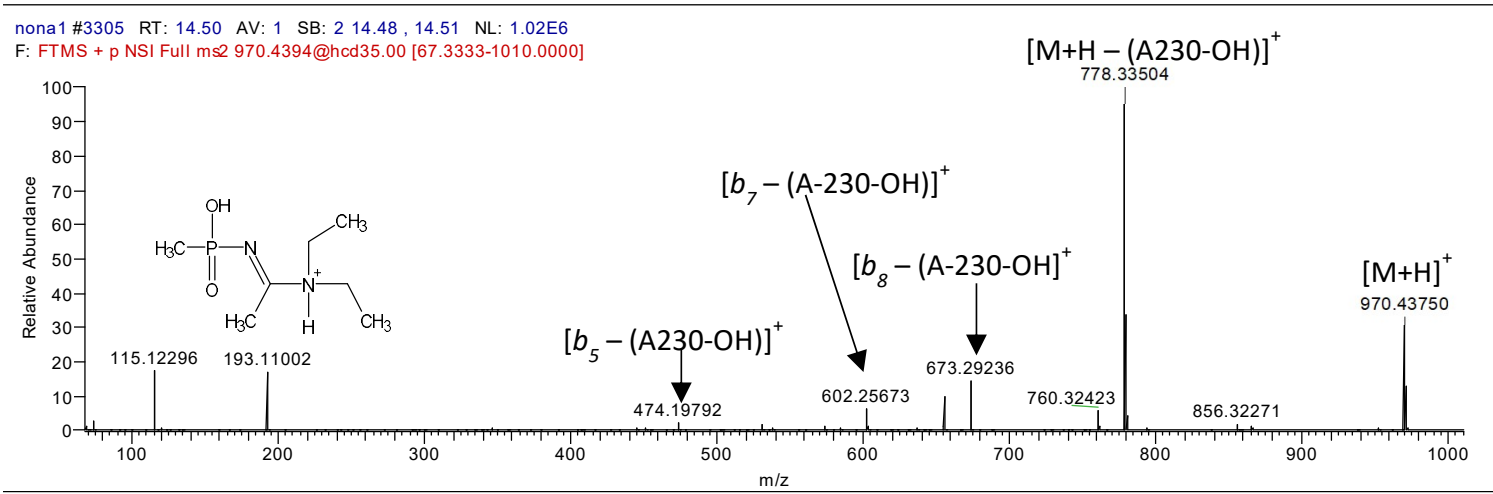

nona1 \#3304 RT: 14.49 AV: 1 SB: $214.47,14.51$ NL: 4.31E6

F: FTMS + p NSI Full ms2 485.7233@hcd20.00 [67.3333-1010.0000]

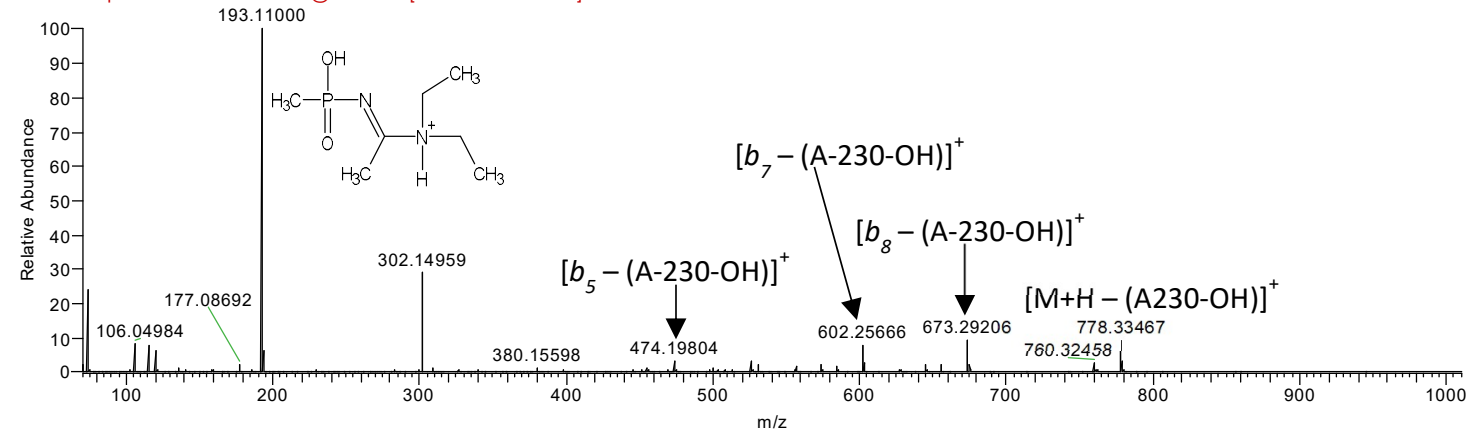

Figure S1. MS/HRMS spectra of the single m/z 970.4394 (upper) and double m/z 485.7233 (lower) protonated molecule of phosphylated nonapeptide derived after pepsin digestion of $\mathrm{HuBuChE}$ isolated from plasma exposed to A-230; the ion with $\mathrm{m} / \mathrm{z} 302.14$ is from a coeluting compound. A-230-OH $=\mathrm{A}-230$ with $\mathrm{F}$ replaced by $\mathrm{OH}$. 
nona1 \#3305 RT: 14.50 AV: 1 SB: 2 14.48, 14.51 NL: 1.76E5

F: FTMS + p NSI Full ms2 970.4394@hcd35.00 [67.3333-1010.0000]

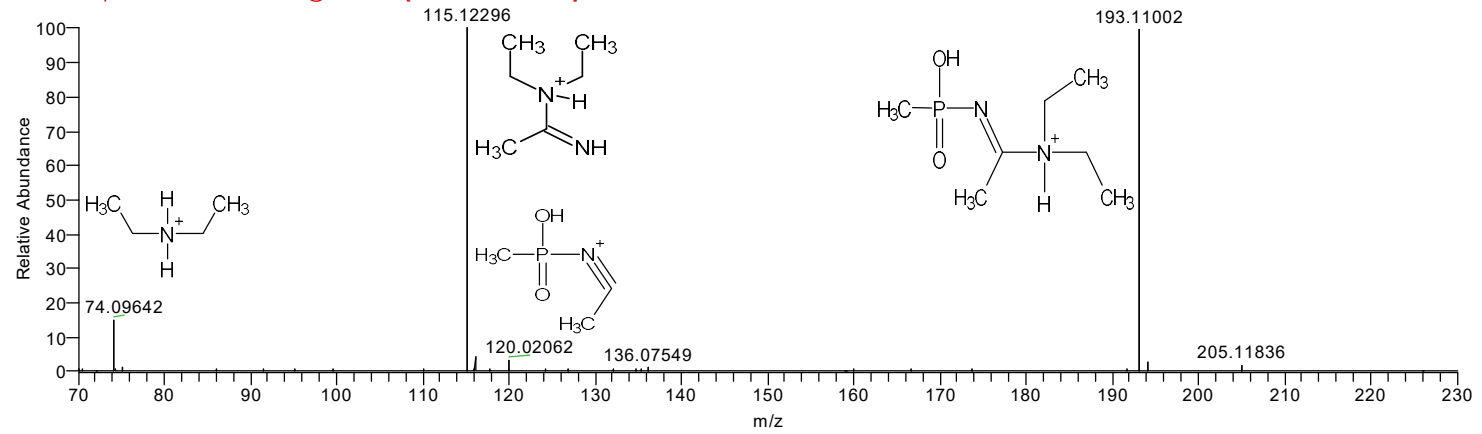

nona1 \#3304 RT: 14.49 AV: 1 SB: 2 14.47, 14.51 NL: 4.31E6

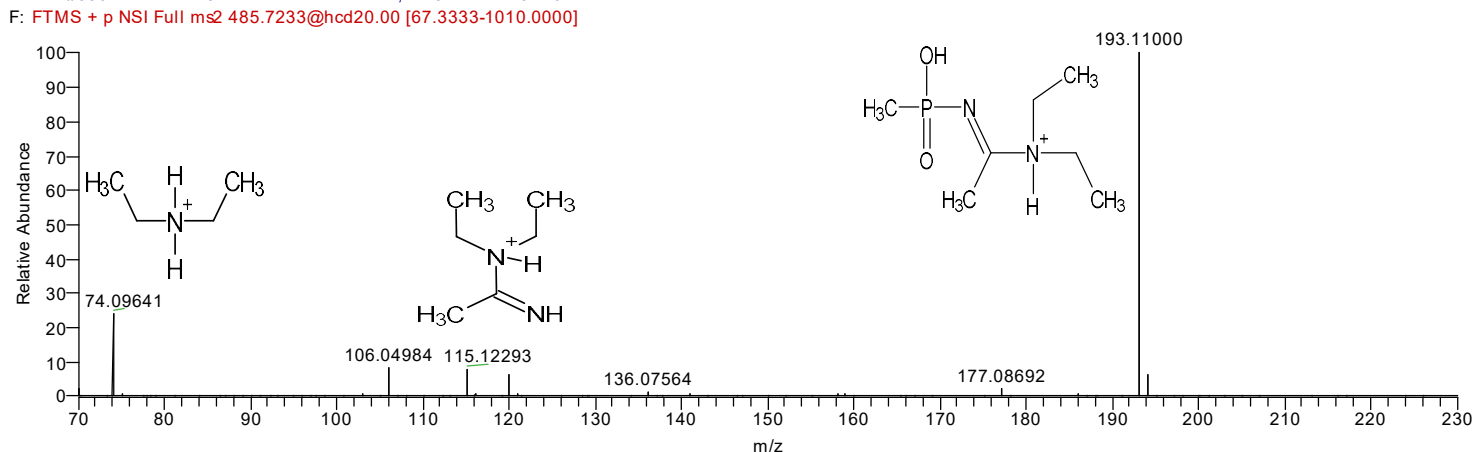

Figure S2. Lower region (m/z 70-230) MS/HRMS spectra of the single m/z 970.4394 (upper) and double $\mathrm{m} / \mathrm{z} 485.7233$ (lower) protonated molecule of phosphylated nonapeptide derived after pepsin digestion of $\mathrm{HuBuChE}$ isolated from plasma exposed to A-230 


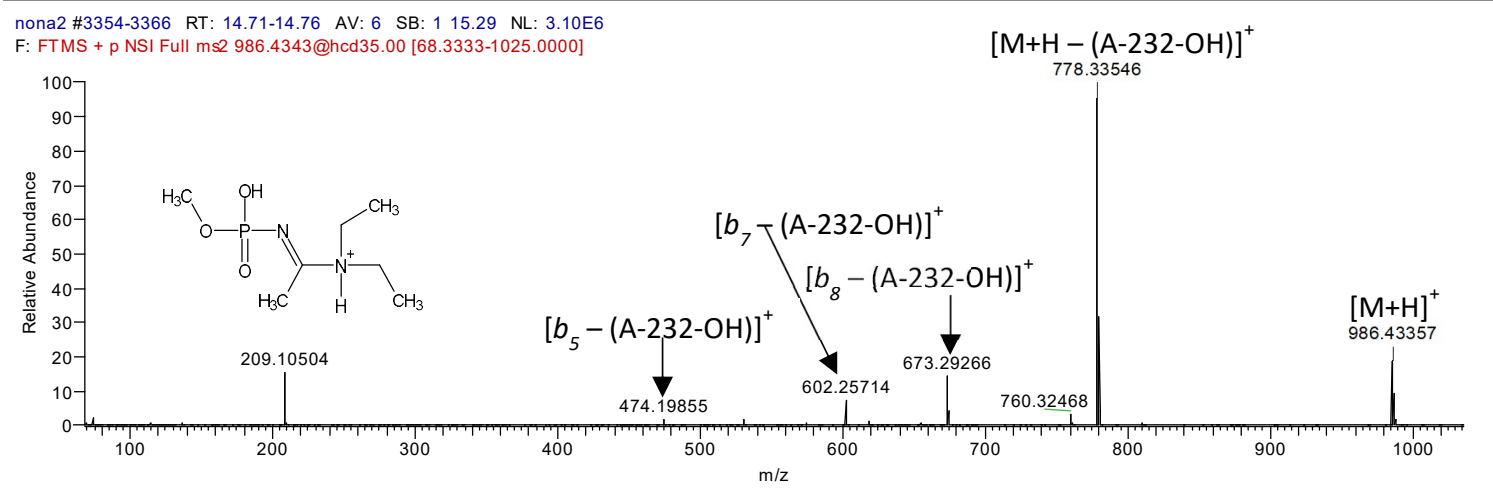

nona2 \#3356-3366 RT: 14.72-14.76 AV: 6 SB: 115.29 NL: 9.67E6

nona2 \#3356-3366 RT: $14.72-14.76$ AV: 6 SB: 115.29 NL: 9.67E6

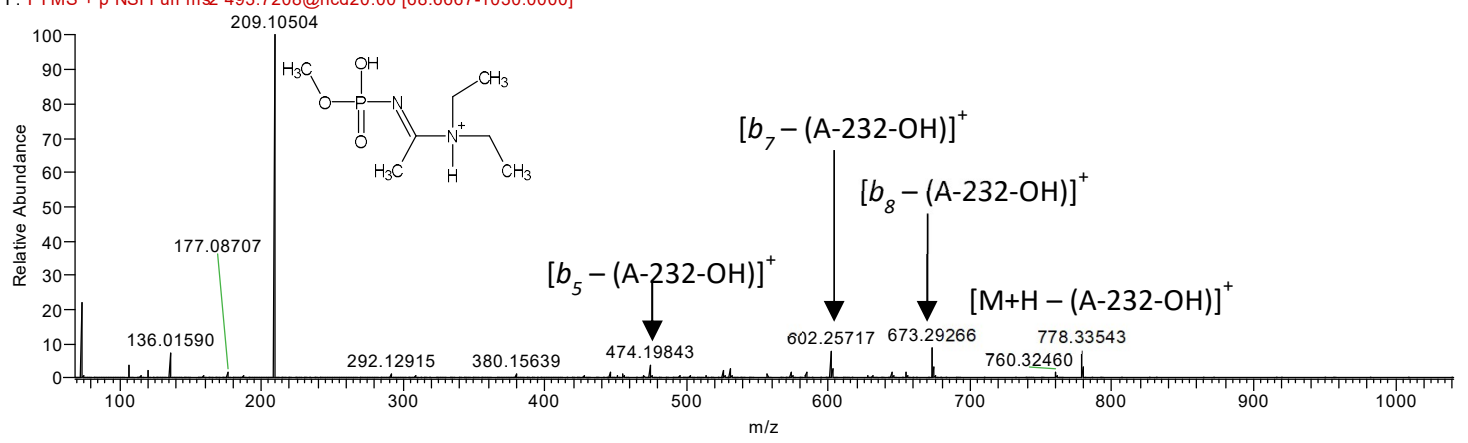

Figure S3. MS/HRMS spectra of the single m/z 986.4343 (upper) and double m/z 493.7208 (lower) protonated molecule of phosphylated nonapeptide derived after pepsin digestion of $\mathrm{HuBuChE}$ isolated from plasma exposed to A-232. A-232-OH $=\mathrm{A}-232$ with $\mathrm{F}$ replaced by $\mathrm{OH}$. 


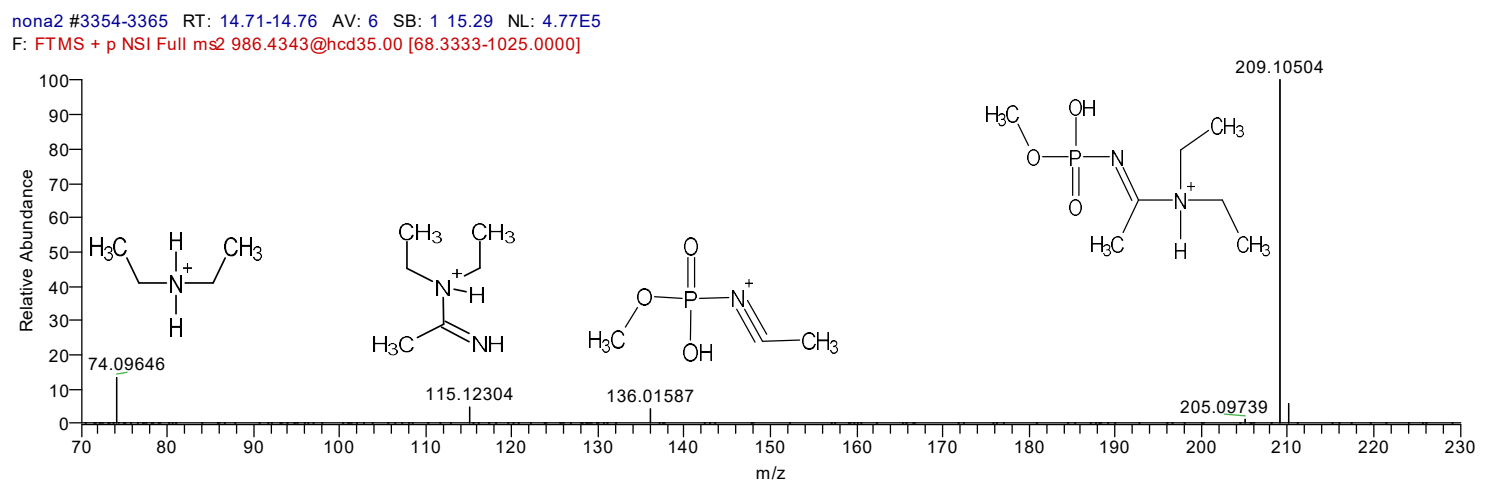

nona2 \#3356-3365 RT: $14.72-14.75$ AV: 5 SB: 115.29 NL: $1.05 E 7$
F: FTMS + p NSI Full ms2 493.7208@hcd20.00 [68.6667-1030.0000]

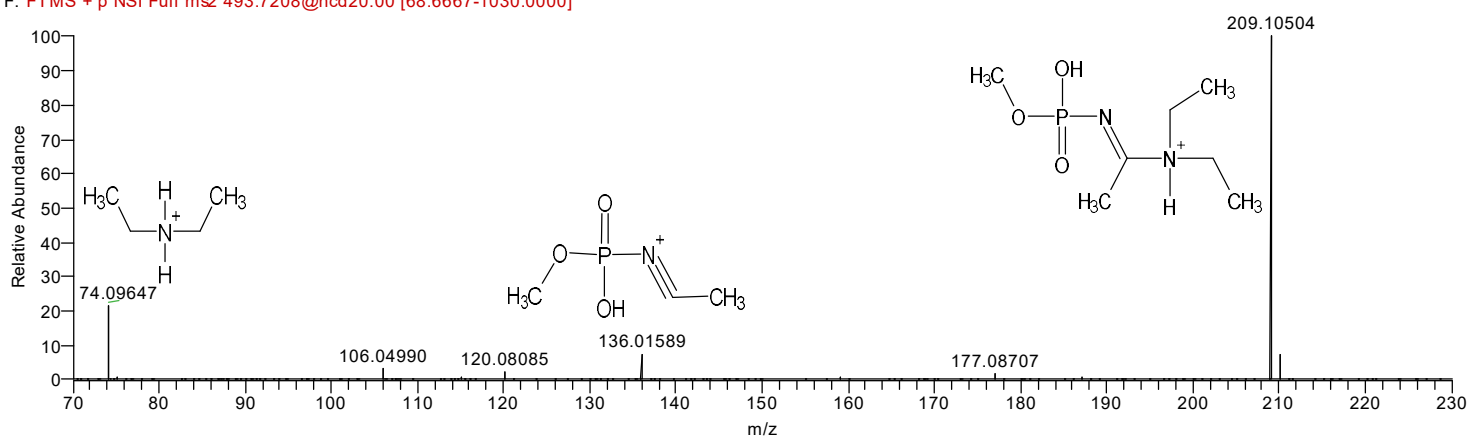

Figure S4. Lower region (m/z 70-230) MS/HRMS spectra of the single m/z 986.4343 (upper) and double m/z 493.7208 (lower) protonated molecule of phosphylated nonapeptide derived after pepsin digestion of $\mathrm{HuBuChE}$ isolated from plasma exposed to A-232 

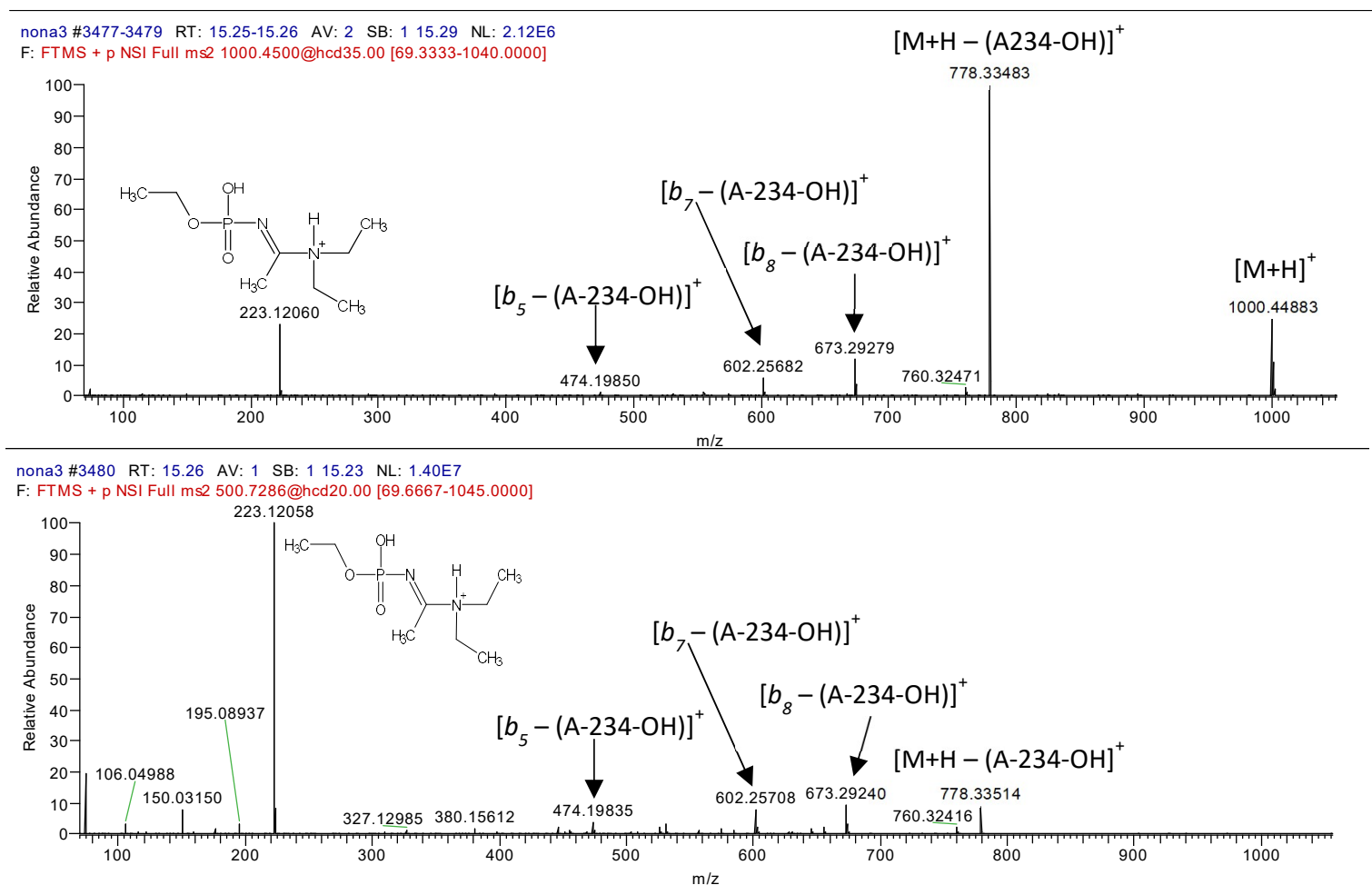

Figure S5. MS/HRMS spectra of the single m/z 1000.4500 (upper) and double m/z 500.7286 (lower) protonated molecule of phosphylated nonapeptide derived after pepsin digestion of $\mathrm{HuBuChE}$ isolated from plasma exposed to A-234. A-234-OH $=\mathrm{A}-234$ with $\mathrm{F}$ replaced by $\mathrm{OH}$. 
nona3 \#3477-3479 RT: 15.25-15.26 AV: 2 SB: 115.29 NL: 4.90E5

F: FTMS + p NSI Full ms2 1000.4500@hcd35.00 [69.3333-1040.0000]

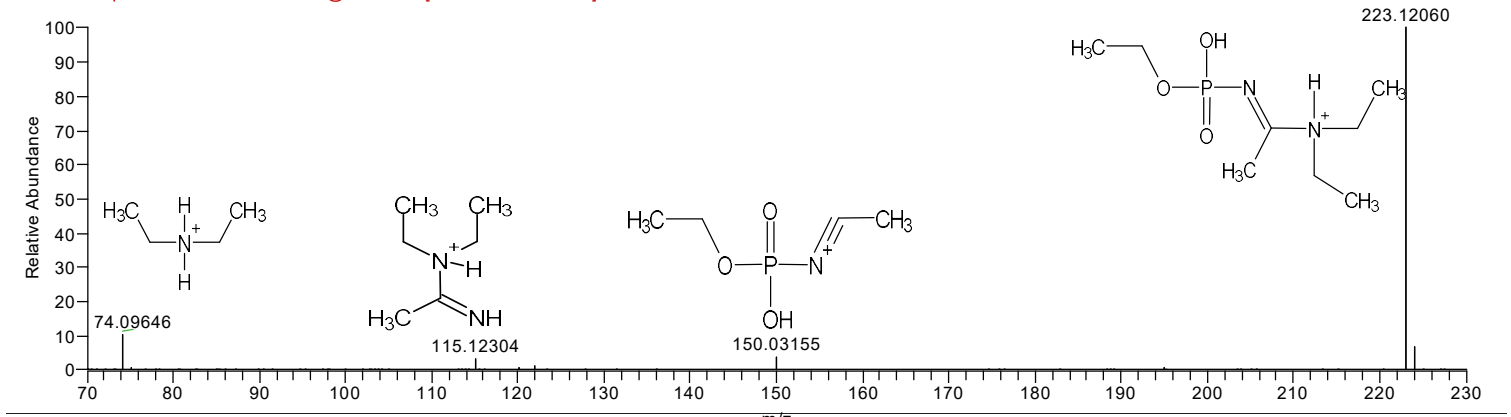

nona3 \#3480 RT: $15.26 \quad$ AV: 1 SB: $115.23 \quad \mathrm{NL}: 1.40 \mathrm{E} 7$

F: FTMS + p NSI Full ms2 500.7286@hcd20.00 [69.6667-1045.0000]

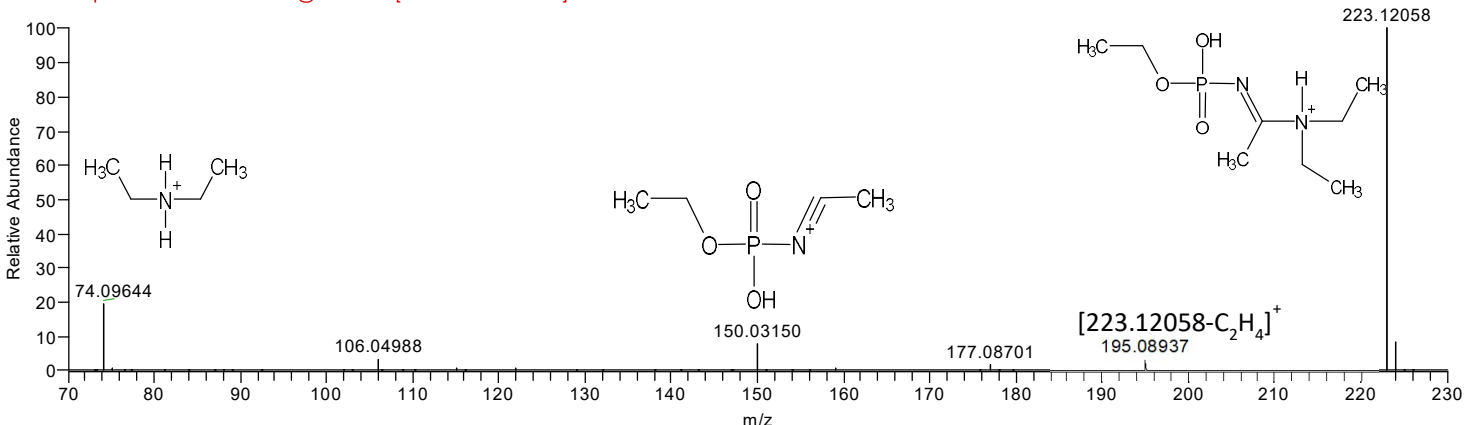

Figure S6. Lower region (m/z 70-230) MS/HRMS spectra of the single m/z 1000.4500 (upper) and double $\mathrm{m} / \mathrm{z} 500.7286$ (lower) protonated molecule of phosphylated nonapeptide derived after pepsin digestion of $\mathrm{HuBuChE}$ isolated from plasma exposed to A-234 